tors, crystals and amorphous layers; photoelectric phenomena in dielectrics and semiconductors; plasma spectroscopy of new gaseous sources of coherent light; Raman effect in crystals and organic molecules by laser excitation; molecular spectroscopy of semiconductors, etc. In the third, where we have grouped devices and integral systems, there are: physics of metal-dielectric-semiconductor structures and their applications in integral microelectronics; physical problems in acoustic electronics; construction of photodiodes, Gunn-diodes, etc.

In addition, as auxiliary methods and instruments, we should mention electron microscope structural investigations; techniques of low temperatures and thermodynamical properties of superconductors, etc.

Physical electronics is, in practice, closely connected with the physics of condensed matter and nuclear physics. The following specific trends in its development can be noted:

Emission and modulation of electron and ion beams: photoelectron and secondary electron emission; electron and ion methods for the formation and treatment of thin films: electron and ion methods for the creation and measuring of high and superhigh vacuum.

Plasma physics: transport phenomena, instability phenomena, interactions with electromagnetic waves, plasma chemistry, voltaic arc, etc.

At superhigh frequences, investigations are being made into gyromagnetic phenomena in radiotranslation elements and sets; applications of semiconducting elements in the creation, amplification and transformation of oscillations ; construction of devices and systems for the transformation of information, etc.

In the domain of quantum electronics, different types of laser (gaseous, metal vapours, dyed liquid) have been constructed, by means of which some questions concerning holography and different non-linear effects have been solved.

Several more general problems such as metrology, methodology, new trends in teaching, etc. are also examined, mainly at the University.

Most of the research work in all branches of physics is carried out in close cooperation with the Joint Insti- tute for Nuclear Research in Dubna, the Centre for Magnetic Phenomena in Wroclaw and other research centres of the socialist countries, as well as in collaboration with Trieste, CERN, Batavia, etc.

We do not consider it necessary to describe here the activities in meteorology, astrophysics, geophysics, biophysics and in other more practical domains of physics or in any border areas with other sciences. It seems to us that the research fields, mentioned above, give an idea of the level, scope and development of physics in Bulgaria. We are carrying out theoretical and experimental research, which is mainly directed at national needs.

We are able to state that physics in Bulgaria is developing well, and results are on a high level, even if research has a relatively limited scope, i.e. of interest for the technical and cultural development of our country. The trend now is to push forward the process of concentration of manpower and material facilities in order to achieve a better connection between the different scientific disciplines and further raise the level of research, training, and application.

\title{
Scattering of Thermal Atoms from Crystal Surfaces
}

\section{G. Benedek, Milan G. Boato, Genoa}

(CNR National Group for the Structure of Materials)
The scattering of neutrals from surfaces is not a new subject. Soon after the first experiments by Dunoyer in 1911, molecular beams were systematically applied by Stern and coworkers in Hamburg to study different phenomena (1919-1933) including scattering from crystal surfaces. The best known result is the first observation of atom diffraction in 1929 - a brilliant confirmation of the wave nature of material particles. We must, however, recall that Stern and coworkers carried out a complete and very accurate study of the diffraction of $\mathrm{He}$ and $\mathrm{H}_{2}$ beams from alkali halide surfaces under different physical conditions. At the same time, Johnson in 1930-31, made similar experiments with a beam of hydrogen atoms. Some of the experimental apparatus used by these workers was so sophisticated, that about forty years elapsed before significantly new experiments in the area of atom scattering from surfaces were carried out.

In the sixties some revival of interest for gas-solid collisions was stimulated by studies in rarefied gas dynamics connected with the launch of satellites. A particular emphasis was given to the collision of molecules in the classical limit, together with problems like the accommodation coefficient and the mean momentum transfer.

An important breakthrough in experimental techniques came from the discovery of supersonic nozzle sources, which provided the means for producing very intense and well collimated quasi-monochromatic beams of atoms and molecules at thermal energies. In the meantime, the whole field of surface physics had started a big expansion, encouraged by the advancing knowledge in solid state science and by the development of more and more refined experimental techniques.

Also the quantum theory of scattering of atoms from surfaces, after the pioneer work of Lennard-Jones and Devonshire in the thirties, made several improvements, starting with the non-perturbative approach suggested by Cabrera, Celli, Goodman and Manson.

\section{Advances in Experimentation}

Starting around 1969-70, several groups in Europe, the USA and $\mathrm{Ca}$ nada, developed new scattering appa- ratus for a more careful study of the interaction of atoms and molecules with surfaces. Particular emphasis was given to the understanding of the coherent scattering from well characterized crystal surfaces; this method of investigation appeared to be complementary to the low energy electron diffraction (LEED) technique for the study of surface structure, and was rich in information about the atom-surface interaction and the surface excitations.

Coherent scattering may be naturally classified into elastic and inelastic diffraction, two subjects which will be briefly reviewed in this article.

In order to observe coherent scattering, several conditions must be fulfilled. To begin with, the de Broglie wave length $\lambda=h(2 m E)^{-1 / 2}$ must be of the same order of magnitude as the surface lattice parameter a. Furthermore, the surface should be ordered over regions of the order of the coherence length of the beam, and thermal motions of the atoms must not be too large. Diffraction is easily observed when beams of light atoms or molecules, e.g. $\mathrm{He}, \mathrm{Ne}, \mathrm{H}, \mathrm{H}_{2}$, and clean cleaved surfaces are used. The 
main features that characterize modern scattering equipment for studying atom diffraction are: intense, narrow and well collimated beams with variable energy and small energy spread; point-like and sensitive detectors; well characterized and long lasting crystal surfaces maintained at temperatures as low as possible; measurements of scattered intensity over a wide angular range, possibly with energy analysis.

The non-hygroscopic alkali halide crystals (particularly LiF, the crystal used by Stern) still constitute the main object of study, owing to the ease of preparation of the surface, and their diffraction strength; however, the number of crystals where diffraction has been observed is now large enough to show the potentiality of the method. In particular, oxides (MgO, $\mathrm{NiO}$, etc.) and metals ( $\mathrm{W}$ and $\mathrm{Ag}$ ) have been the subject of recent studies.

\section{Elastic Diffractive Scattering}

The angular position of diffraction peaks relative to the incident beam is directly related, as in LEED, to the surface structure, through the conservation of both energy and parallel "crystal momentum". The diffraction figure, however, reflects only the structure of the topmost layer of the crystal surface, owing to the lack of penetration of neutral particles at thermal energies.

The intensity of diffraction peaks is related to the periodic part of the atom-surface potential. Excluding chemical interactions, this potential consists of a short range strongly repulsive part and a longer range weakly attractive part. It is the repulsive part that contributes mostly, through its periodicity strength, to the distribution of diffracted intensity; so we expect that the intensity pattern gives us information on the "corrugation" (at atomic scale) of the crystal surface. This prediction is confirmed by the experimental results and by theory.

As an example of a strongly corrugated surface, in Fig. 1 (a) we show the diffraction pattern of He scattered by LiF (001) at normal incidence; similar patterns are shown by the systems $\mathrm{Ne}-\mathrm{LiF}$ (001), He-NaF (001), and $\left.\mathrm{H}-\mathrm{KCl}(001)^{1-3}\right)$. In all these cases, the scattered intensity is distributed over a large angle and among a large number of diffraction peaks. The intensities of high-order peaks may be larger than those of low-order peaks, (00) specular peak included. The corrugation found in several alkali halide (001) surfaces is due to the alternation of large negative and small positive closed-shell ions, which results in a surface having roughly a sinusoidal shape in two dimensions. This is confirmed by theory. A hard surface model, with a shape function given by:

$z=1 / 2 \zeta_{0}(\cos 2 \pi x / a+\cos 2 \pi y / a)$

treated in the framework of quantum mechanical scattering theory, is able to predict with an accuracy of a few percent the intensity of the diffraction peaks observed for the system He-LiF and $\mathrm{Ne}-\mathrm{LiF}^{4}$ ). The value found for $\zeta_{0}$ is about $0.30 \AA$. Improvement to this model can be obtained by adding higher harmonics to expression $\left.(1)^{5}\right)$.

In contrast to alkali halides, metal surfaces (particularly if densely packed) appear to be very flat as seen by the incident neutral particles. Experiments carried out in past years on a number of metal surfaces had yielded only a specular peak, with the exception of the special (112) face of tungsten, corrugated in one dimension. Only recently, by using a very high sensitivity and a low surface temperature, first-order diffraction peaks were observed in $\mathrm{Ag}$ (111), see Fig. 1 (b). Their intensity is about $10^{-3}$ times that of the specular peak, showing a very small amplitude of surface corrugation; this is indeed expected, owing to the smoothing effect of conduction electrons.

More accurate and systematic measurements of the elastic diffraction probabilities, carried out on a more numerous set of gas-surface systems and combined with more refined theoretical treatments, should eventually lead to detailed information on the atom-surface potential; the technique is capable of becoming complementary to LEED for the investigation of the surface structure of the topmost layer of crystal faces.

\section{Bound State Resonances}

Another important effect, which contains further interesting information on the attractive part of the atom-surface potential, is the resonant scattering. This is due to the possible existence of resonant bound states with positive energy. In practice, the incoming atom, for certain values of energy $E_{i}$ and incidence angle $\Theta_{\mathrm{i}}$, can be captured in a resonant bound state of the surface for a time long enough to interact several times with crystal vibrations (selective adsorption). In this case the atom can be re-emitted in a state having no correlation with the incident state, and is lost to all effect. Therefore, when the incident beam fits the resonant condition, a dip usually appears in the intensity of the specular and other diffraction peaks, Fig. 2(a).
This phenomenon, discovered as early as 1933 by Frisch and Stern and interpreted three years later by Lennard-Jones and Devonshire, is now the subject of several experimental ${ }^{1-3}$ ) and theoretical ${ }^{6}$ ) investigations, whose purpose is a better description of the gas-surface potential well. The example of Fig. 2(b) shows the atomsurface potential well for the system $\mathrm{H}(\mathrm{D})$ - NaF (001) as deduced by the Erlangen group ').

The physical basis of the resonant scattering is quite simple. The quantum levels of an atom subject to a periodical surface potential form a band structure. According to Bloch's theorem the eigenstates are $2 \mathrm{D}$ wavelike combinations of wavevector $\mathbf{K}$ of the localized orbitals of each potential well, the latter being classified by the quantum numbers $N$ (excitations along the surface) and $n$ (excitations normal to the surface). Usually, the few lowest values of $n$ correspond to bound states localized at the surface; nevertheless, for large values of the kinetic energy parallel to the surface (large $N$ ), the energy $E_{N n}(\mathbf{K})$ becomes positive. This eigenvalue, falling into the free atom continuum spectrum, yields a resonance. More precisely, this occurs when $E=E_{N n}(\mathbf{K})$ and the surface component of the incident wavevector equals $\mathbf{K}$ up to a surface reciprocal lattice vector.

The interpretation of the experimental resonances is commonly based on the quite crude assumption of a free motion of the atom along a flat surface, and well defined bound state energies $E_{n}<0$ for the motion normal to the surface. Only recently Chow and Thompson have abandoned this approximation, predicting interesting band-structure effects due to the surface periodicity ${ }^{7}$ ).

\section{Inelastic Processes and Spectroscopy of Surface Phonons}

Two types of inelastic diffraction processes are possible in the gassurface scattering. The first one is connected with the exchange of energy among internal degrees of freedom of the colliding molecule (e.g. the recently observed rotationally inelastic transitions during the collision of hydrogen molecules with surfaces). The second one is the inelastic process involving creation or annihilation of crystal phonons.

Here we shall concentrate particularly on this second aspect, as these processes are far and away the most frequent events, the elastic scattering being in several cases only a 

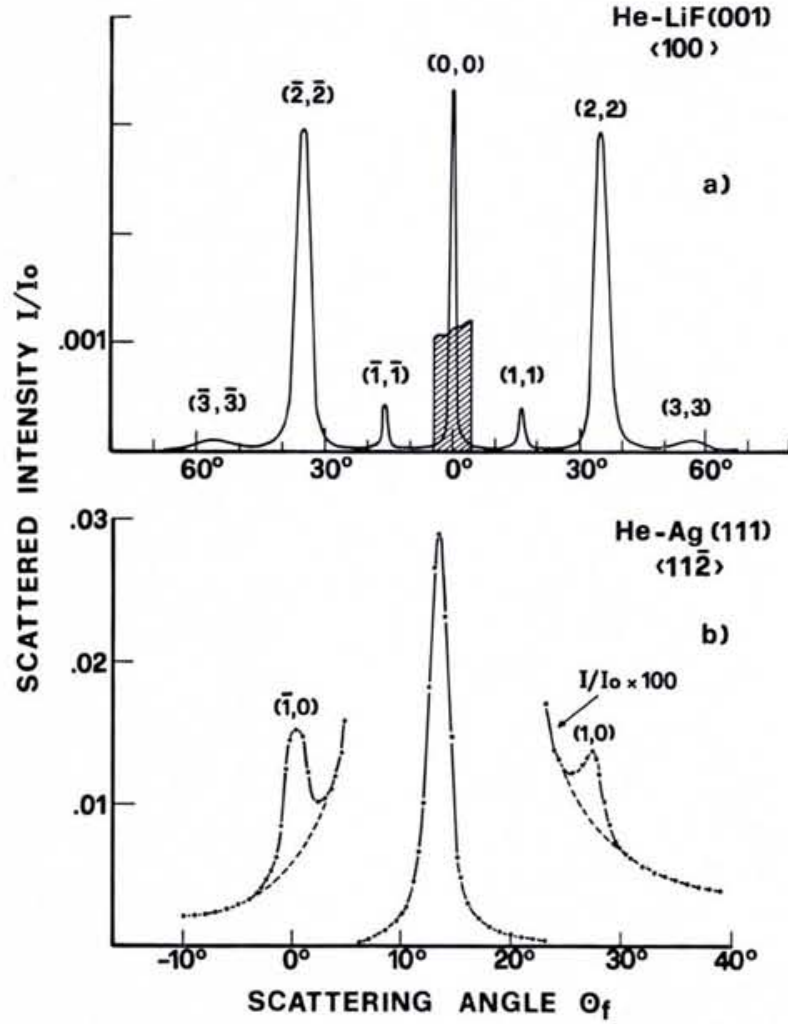

Fig. 1 Diffraction of thermal $\mathrm{He}$ atoms $\left(E_{i} \sim 63 \mathrm{meV}\right)$ from crystal surfaces: a) LiF (001) at normal incidence, in the $\langle 100\rangle$ azimuthal plane; b) $\mathrm{Ag}$ (111) at $\Theta_{i}=13.5^{\circ}$ in the $\langle 112\rangle$ azimuthal plane. The surface temperature was $80^{\circ} \mathrm{K}$ in both cases. (From Ret. 2 and from Boato, Cantini and Tatarek, J. Phys. F., 6 (1976) L237).

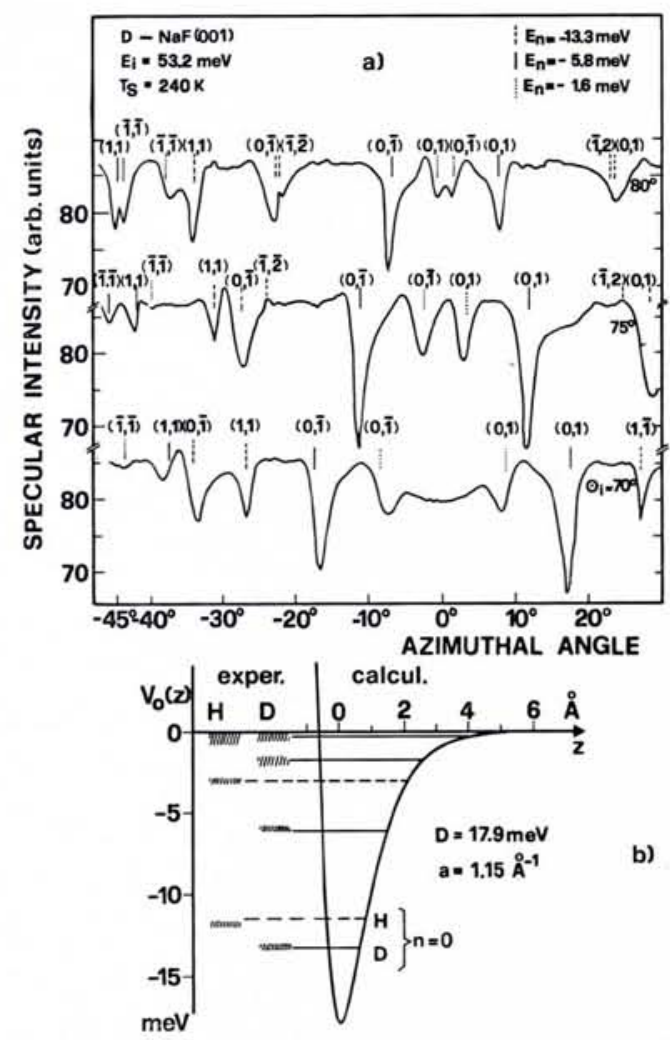

Fig. 2 Selective adsorption in the system atomic hydrogen sodium fluoride: a) Bound state resonance dips in the specular intensity as a function of the azimuthal angle for three different values of $\Theta_{i}$; b) The atom-surface potential derived from bound state resonances, using both hydrogen and deuterium as incident atoms. $D$ is the well depth and a the inverse length repulsive parameter (Ref. 1 ). small contribution ${ }^{8}$ ). Inelastic processes received much attention because of their role in the accommodation coefficient and all gas-solid energy transfers. The experiments carried out by Subbarao and Miller $^{9}$ ) with very low energy $\mathrm{He}$ beams on $\mathrm{Ag}$ (111) provided a first clear separation between elastic and inelastic scattering.

The inelastic scattering contains first of all information on the mean square vibrational displacement of surface atoms via the Debye-Waller factor ${ }^{10}$ ). Furthermore, Williams, through sophisticated measurements of the angular distribution of $\mathrm{He}$ from $\mathrm{LiF}$ and $\mathrm{NaF}$, was able to show that the streaks found around the elastic peaks might correspond to one-phonon processes and, in some cases, can be associated with Rayleigh waves $\left.{ }^{3}\right)$. Indeed, since the theoretical work of Cabrera, Celli and Manson in 1969, the great potentialities of the inelastic scattering studies for the direct detection of surface vibrations were apparent. This is perhaps the most appealing application of the nozzle beam experiments because of the specific importance of surface phonons.

Surface phonons are involved in several surface phenomena as well as in many interesting technical applications, among which we can mention heat transmission through interfaces, surface faceting, segregation and fracture, the analysis of adsorbates, the use of surface waves in ultrasonic delay lines and signal processing devices. However, in spite of their wider importance, the experimental knowledge of surface vibrations in the dispersive region $(\mathrm{GHz}$ to $\mathrm{THz})$ is distinctly sparser than that of bulk vibrations, because the conventional techniques, such as neutron, infrared and Raman spectroscopies, meet severe limitations in the study of surface phonons in monocrystals.

It is obvious that non-penetrating beams are more suitable probes for surface phonons. Electron energy loss spectroscopy has given us the first clear evidence of surface excitations in monocrystals "), but this method is restricted to very long-wave phonons $(K \sim 0)$, due to the small electron mass. For this reason, the inelastic scattering of thermal atoms seems today, to be the only practicable way of revealing surface phonons for $\mathbf{K}$ ranging all over the surface Brillouin zone.

The study of surface dynamics seeks to identify, first of all, the frequencies of surface localized modes for each $\mathbf{K}$ value, namely the surface mode dispersion relations, and, second, the surface density of phonon states for each $\mathbf{K}$ value. Inelastically reflected atoms carry, in principle, all this information, provided that joint accurate analysis of both velocity and angular distributions of the scattered atoms is made. For example, the velocity distribution of atoms scattered normally to the surface would give just the energy-loss spectrum for a fixed surface momentum transfer $\mathbf{K}$, the latter varying with the incidence angle. Since, at sufficiently low beam energies, the one-phonon processes are predominant, one would expect the energy-loss spectrum be just the spectral density of surface phonons. Unfortunately the situation is less simple. The incident atom interacts with different phonons with a different strength : in other words, the energyloss spectrum reflects the phonon density in a quite distorted way.

How large is such distortion? The answer to this question lies in the calculation of a form factor relating the energy-loss profile to the surface phonon density. A first example of such a calculation was reported in 1974 for the system He-LiF $(001)^{12}$ ); 
it is shown in Fig. 3, where the surface phonon density for $\mathbf{K}$ at the border of the surface Brillouin zone is compared with the theoretical energyloss spectrum. Clearly the form factor introduces an enhancement in the response of acoustic phonons (mainly Rayleigh waves $S_{1}$ ) and a dramatic cut-off in the optical region, where only the sagittal Lucas mode $\left(\mathrm{S}_{4}\right)$ survives. This result is not unexpected for neutrals interacting with crystal ions only via Lennard-Jones forces, because these forces act nearly in phase on the ions, regardless of their charge sign.

One could argue that any future advance in the surface phonon study from combined angular and time-offlight analysis of the scattered beam, requires a joint progress in the theory of the form factor. This is, regrettably, a quite optimistic view, since the coupled high-resolution measurements of both velocity and angular distribution are still very hard to do, owing to the difficulty of improving three simultaneous properties in a single detector: sensitivity, speed and smallness.

However, the present difficulties have stimulated interesting refine-

Fig. 3 The surface phonon density of LiF (001) for $\mathbf{K}$ at the border of the surface Brillouin zone, compared with the theoretical energy-loss spectrum taken for normal scattering (Ref. 12). The scattering displays an enhanced response of the Rayleigh wave $\left(S_{1}\right)$ and a cutt-off in the optical region, where only the sagittal Lucas mode $\left(\mathrm{S}_{4}\right)$ survives. In the phonon spectrum, the Rayleigh wave represents $15 \%$ of the integrated density, while in the energy-loss spectrum its contribution rises to $50 \%$.

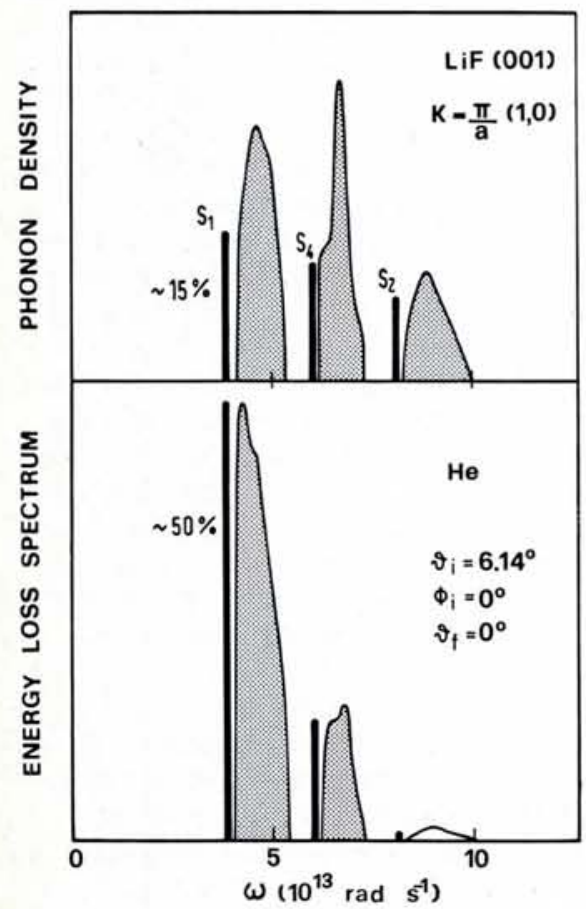

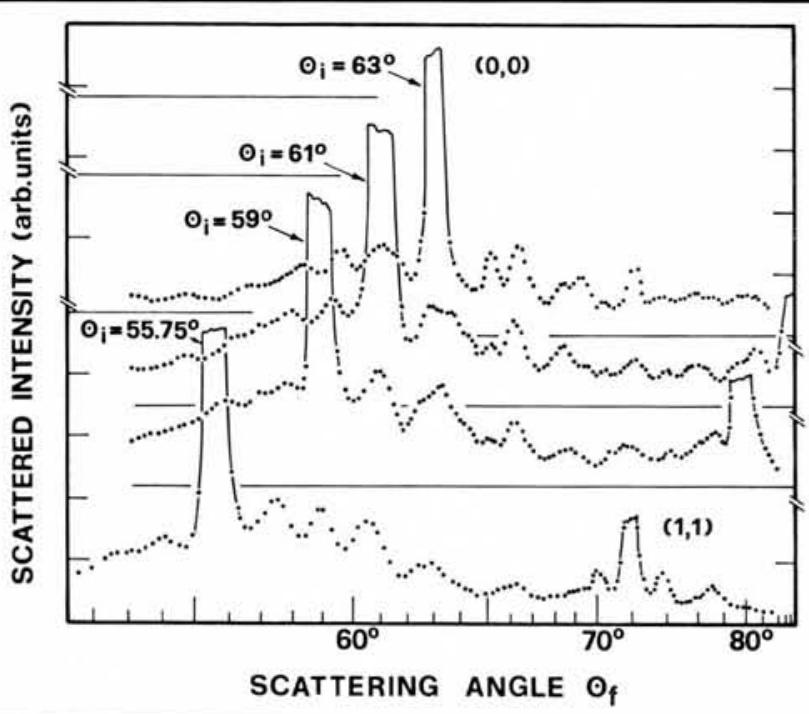

Fig. 4 Structures in the inelastic background around diffraction peaks in the scattering of Ne from LiF (001), at different incident anales $\Theta_{i}$ The dips are believed to be due to inelastic resonant transitions (Cantini, Felcher and Tatarek, to be published).

ments in the study of angular spectra with special attention to the structures appearing between the diffraction peaks, in order to see whether it is possible to extract some information on surface dynamics without a timeof-flight analysis. Indeed, William's work has made way for the study of more complicate inelastic spectra, like those found with $\mathrm{Ne}$ beams in Genoa. The scattering of $\mathrm{Ne}$, almost completely inelastic, yields several well resolved structures between the diffraction peaks, which seem to form as a whole a difficult puzzle, Fig. 4.

In a recent work ${ }^{13}$ ), it was shown that the main peaks of the angular distribution could be associated with analytical critical points of the surface phonon dispersion curves, particularly those of Rayleigh and Lucas modes. As a consequence, the frequencies and wavevectors of these modes could be measured by analyzing the shift of the peaks under a change of the incidence angle. But things do not seem to be so simple: this experiment would need in principle a better beam monochromaticity than that achieved till now in most experiments. Furthermore, just a few months ago Cantini, Tatarek and Felcher, in attempting this kind of measurement, made another interesting discovery: several dips of the angular distribution can be interpreted as due to inelastic resonant transitions, and again, with a procedure similar to that of Williams, the Rayleigh dispersion curve can be derived ${ }^{14}$ ).

In conclusion, such a large variety of phenomena influencing the scattering of thermal atoms, at the present time makes the disentanglement of the angular distributions quite hard, without a time-of-flight analysis. On the other hand, as this is due to their high information content, the full reading of angular spectra poses a fascinating problem. Hopefully, we look for an answer to the challenge presented: the spectroscopy of surface phonons.

\section{REFERENCES}

1. FINZEL, H.U., FRANK, H., HOINKES, $H$., LUSCHKA, M., NAHR, H., WILSCH, H. and WONKA, U., Surf. Sci. 49 (1975) 577.

2. BOATO, G., CANTINI, P. and MATTERA, L., Surf. Sci. 55 (1976) 151.

3. WILLIAMS, B.R., J. Chem. Phys. 55 (1971) 1315 and 3220 .

4. GARIBALDI, U., LEVI, A.C., SPADACINI, R. and TOMMEI, G.E., Surf. Sci. 48 (1975) 649.

5. GARCIA, N., IBANEZ, J., SOLANA, J. and CABRERA, N., Surf. Sci. 60 (1976) 385 6. TSUCHIDA, A., Surf. Sci. 14 (1969) 375 GOODMAN, F.O., Surf. Sci. 19 (1970) 93 ; CABRERA, N., CELLI, V., GOODMAN, F.O. and MANSON R., Surf. Sci. 19 (1970) 67. 7. CHOW, H. and THOMPSON, E.D., Surf. Sci. 59 (1976) 225 ; these effects have been recently observed by LIVA, M.P., DERRY, G. and FRANKL, D.R., Phys. Rev. Letters 37 (1976) 1413.

8. An interesting recent discussion is found in LIN, Y.W. and WOLKEN, G., J. Chem. Phys. 65 (1976) 2634. A good review article is SMITH, J.N. Jr., Surf. Sci. 34 (1973) 613

9. SUBBARAO, R.B. and MILLER, D.R., J. Chem. Phys. 51 (1969) 4679.

10. BEEBY, J.L., J. Phys. C 4 (1971) L 359 ; ARMAND, G., LAPUJOULADE, J. and LEJAY, Y., J. Physique Lettres 37 (1976) 187 ; COMSA, G., J. Phys. C, 6 (1973) 2648.

11. IBACH, H., Phys. Rev. Letters, 27 (1971) 253.

12. BENEDEK, G. and SERIANI, G., Jpn. J. Appl. Phys., Suppl. No. 2, Pt. 2 (1974) 545

13. BENEDEK, G., Phys. Rev. Letters, 35 (1975) 234

14. CANTINI, P., FELCHER, G.P. and TATAREK, R., Phys. Rev. Letters, 37 (1976) 606. 